\title{
DU PASSÉ FAISONS TABLE RASE ?, UN VANDALISME ÉPISTÉMOLOGIQUE ? \\ Vincent Chambarlhac
}

Centre d'histoire de Sciences Po | «Histoire@Politique »

2014/2 n²3 | pages 191 à 203

Article disponible en ligne à l'adresse :

https://www.cairn.info/revue-histoire-politique-2014-2-page-191.htm

\section{Pour citer cet article :}

Vincent Chambarlhac, «Du passé faisons table rase ?, un vandalisme épistémologique ? », Histoire@Politique 2014/2 (n²3), p. 191-203. DOI 10.3917/hp.023.0191

Distribution électronique Cairn.info pour Centre d'histoire de Sciences Po.

(C) Centre d'histoire de Sciences Po. Tous droits réservés pour tous pays.

La reproduction ou représentation de cet article, notamment par photocopie, n'est autorisée que dans les limites des conditions générales d'utilisation du site ou, le cas échéant, des conditions générales de la licence souscrite par votre établissement. Toute autre reproduction ou représentation, en tout ou partie, sous quelque forme et de quelque manière que ce soit, est interdite sauf accord préalable et écrit de l'éditeur, en dehors des cas prévus par la législation en vigueur en France. Il est précisé que son stockage dans une base de données est également interdit. 
Vincent Chambarlhac, «Du passé faisons table rase?, un vandalisme épistémologique?», Histoire@Politique. Politique, culture, société, $\mathrm{n}^{\circ} 23$, mai-août 2014 [en ligne, www.histoirepolitique.fr]

\title{
Du passé faisons table rase?, un vandalisme épistémologique?
}

\author{
Vincent Chambarlhac
}

Antoine Prost note la centralité, aujourd'hui perdue, de l'histoire ouvrière durant l'entre-trois-mai ${ }^{1}$. L'histoire ouvrière conquiert sa légitimité universitaire ; une revue, Le mouvement social, l'illustre en pratiquant une histoire scientifique, une histoire sociale du mouvement ouvrier. Ce mouvement coïncide, autour de 1974, avec le questionnement du Faire de l'histoire par Michel de Certeau, par la Nouvelle histoire $^{2}$. Dans le champ de l'histoire ouvrière, naguère militante, la question d'une pratique scientifique, objectivée, trace de nouvelles frontières entre ce qui relève de logiques scientifiques et ce qui s'en démarque, par l'engagement explicite de l'historien. Le jeu des comptes rendus, le heurt de revues aux objets communs mais aux dynamiques irréductibles les unes aux autres, offrent un lieu pour questionner cette nouvelle frontière où, hors du périmètre de l'histoire scientifique, campent des historiens dont l'engagement assumé interpelle. Prenant l'argument d'un « vandalisme épistémologique » pour se saisir de la réception de ces travaux en regard de revues davantage inscrites dans le champ scientifique, il s'agira ici d'interroger les liens de l'histoire ouvrière scientifique à l'histoire militante. La réception par les Annales de l'ouvrage de J ean Chesneaux, Du passé faisons table rase ?33, paru en 1976, introduit le propos, s'arrimant d'emblée à un questionnement structurant: qu'est-ce qu'une bonne pratique historique à l'heure où triomphe la Nouvelle histoire? Deux lignes suffisent au rédacteur anonyme des Annales pour statuer sur l'ouvrage :

«Jdanov à Pékin? Un retour feutré, mais maoïste, à la dichotomie "science bourgeoise, science prolétarienne" ${ }^{\prime \prime}$. »

Dans l'art délicat du compte rendu, le rédacteur exerce là une forme de police sur la circulation et la réception des ouvrages ${ }^{5}$. Le propos est à double détente. Son anonymat engage l'ensemble du collectif de la revue; sa concision exclut Du passé faisons table rase? de l'ordre scientifique du livre en histoire, renvoyant l'essai à son statut militant. Lequel se signifie par «J danov à Pékin », soit une référence explicite

\footnotetext{
${ }^{1}$ Antoine Prost, «La centralité perdue de la classe ouvrière », dans Autour du Front populaire. Aspects du mouvement social au XXe siècle, Paris, Seuil, coll. « L'Univers historique », 2006, p. 7-16.

2 J acques Le Goff, Pierre Nora, Faire de l'histoire, trois tomes, Paris, Gallimard, 1974. Michel de Certeau, L'écriture del'histoire, Paris, Gallimard, 1975.

${ }^{3}$ J ean Chesneaux, Du passé faisons table rase? À propos de l'histoire et des historiens, Paris, Maspéro, 1976.

${ }^{4}$ Annales. Économie, Sociétés, Civilisations, année 1976, n² 2, p. 464.

5 Bertrand Müller, «Critique bibliographique et construction disciplinaire: l'invention d'un savoirfaire », Genèses, n 14, 1994.
} 
Vincent Chambarlhac, «Du passé faisons table rase?, un vandalisme épistémologique?», Histoire@Politique. Politique, culture, société, $\mathrm{n}^{\circ} 23$, mai-août 2014 [en ligne, www.histoirepolitique.fr]

au long article éponyme que publie Emmanuel Le Roy Ladurie pour le supplément littéraire du Monde ${ }^{6}$. La plume du secrétaire des Annales, qui dirige les pages histoire du quotidien depuis 1972, se charge ainsi du réquisitoire politique : l'horizon de la neutralité est la condition sine qua non, non d'une histoire scientifique, mais plus exactement de la péjoration d'entreprises militantes dans le domaine de l'histoire. Une seconde citation informe à nouveau ce constat :

« Des analyses solidement marquées du sceau de l'idéologie qui ont parfois le charme de la bande-dessinée, mais un goût très sain du document et de bonnes pistes de recherches, sur le travail des enfants au XIX ${ }^{\circ}$ siècle et les instituteurs-artisans face à la politique de Guizot ${ }^{7}$. »

Les Annales commentent ainsi en 1977 la troisième livraison de la revue Les Révoltes logiques, elle-même issue d'un collectif maoïste, qui entend travailler au dévoilement d'une autre mémoire, et prend le XIX ${ }^{\mathrm{e}}$ siècle ouvrier comme objet d'étude. Fondée à l'hiver 1975 autour d'une équipe animée par J acques Rancière, éditée chez Solin ${ }^{8}$, la revue entend proposer une histoire de la révolte, réinterrogeant deux siècles d'histoire militante ${ }^{9}$. En partie venue de l'althussérisme de Mai 68, le collectif considère alors que l'histoire éclaire les enjeux du présent, qu'elle est ainsi politique ici et maintenant. Si elle est proche d'une part des thématiques avancées par Michel Foucault - et notamment la question du pouvoir et des micro-pouvoirs - dans son questionnement de l'archive, la revue se donne d'autres objectifs. D'abord centré sur une « mémoire populaire des luttes » qu'il faudrait retrouver, le projet initial de la revue mute rapidement au profit d'un questionnement sur l'altérité. Les Révoltes logiques ne survivent pas à l'entre-deux-mai, la thèse de J acques Rancière, La nuit des prolétaires, en constitue le palimpseste et en condense les propositions ${ }^{10}$. Elle ouvre d'autres voies, selon Antoine Prost, à l'histoire ouvrière ${ }^{11}$. Pour les Annales, la revue peut trouver sa place dans l'ordre scientifique du livre par son appétence documentaire quand, dans le même mouvement, l'idéologie vaut réserve, péjoration d'un récit ramené à l'histoire de France en bandes-dessinées qui paraît alors chez Larousse ${ }^{12}$.

Que disent ces citations? Elles informent sur le regard des Annales, revue structurante et déterminante dans le champ historique sur les marges de l'institution, entre pratique de l'histoire et engagement militant. C'est dans l'ordre implicite du faire de l'histoire et des règles du métier qui l'enchâssent qu'il faut saisir les productions chroniquées. L'argument du vandalisme - implicite dans la virulence des comptes rendus - trouve ici toute son efficacité heuristique. Au plus près du sens que

\footnotetext{
6 Emmanuel Le Roy Ladurie, «J danov à Pékin », Le Monde, 14 mai 1976.

${ }^{7}$ Annales, Économie, Sociétés, Civilisations, année 1977, volume 32, p. 4.

8 Vincent Chambarlhac, « "Nous aurons la philosophie féroce”. Les Révoltes logiques (1975-1981) », La Revue des revues, $n^{\circ} 49$, printemps 2013.

${ }^{9}$ J acques Rancière, « Les gros mots », dans Les Scènes du peuple, Paris, Horlieu éditions, 2003.

${ }^{10} \mathrm{~J}$ acques Rancière, « Et tant pis pour les gens fatigués !, Entretien réalisé par Edmond El Maleh » pour Le Monde en 1981, dans le recueil : Jacques Rancière, Et tant pis pour les gens fatigués ! Entretiens, Paris, éditions Amsterdam, 2009, p. 41.

11 Antoine Prost, op. cit., p. 13.

12 La première livraison de l'Histoire de France en bandes-dessinées (de Vercingétorix aux Vikings) débute en 1976. Les livraisons s'échelonnent jusqu'en 1978.
} 
Vincent Chambarlhac, «Du passé faisons table rase?, un vandalisme épistémologique?», Histoire@Politique. Politique, culture, société, $\mathrm{n}^{\circ} 23$, mai-août 2014 [en ligne, www.histoirepolitique.fr]

lui conférait l'Abbé Grégoire, il implique la tension entre un patrimoine jugé menacé (une manière de faire de l'histoire scientifique) et sa destruction à des fins où l'historien est politique, la volonté de faire table rase. De manière plus ample, et bien que le mot lui-même ne soit jamais utilisé par les protagonistes, j'entends dans le mot «vandale » l'extériorité d'une pratique de l'histoire à sa définition académique, réassurée par le succès des Annales comme par l'essor de l'histoire scientifique du mouvement ouvrier autour du Mouvement social. Dans le contexte de 1976, entendues dans leur rapport à l'histoire comme métier et science, les citations des Annales participent implicitement d'un tel type de regard. Elles ont à voir avec la question du mouvement communiste: le groupe 69 (Le Roy Ladurie, Le Goff, Burguière...) qui a pris la direction des Annales souhaite faire une revue « de recherche de pointe », et dans cette redéfinition glisse vers un « anticommunisme de gauche» selon Marc Ferro ${ }^{13}$. Ce glissement discret sur le versant politique correspond dans l'ordre du projet à une revue moins ouverte sur l'ensemble des champs de l'histoire, abandonnant là peu à peu des rubriques tangentielles au rôle social de l'historien, comme enseignant notamment. L'accusation implicite de vandalisme, adossée au fait communiste, se supporte d'autant plus que le titre même de l'essai de Jean Chesneaux est un emprunt à l'Internationale, rédigée aux lendemains d'une Commune dont le souvenir transparaît aussi dans Les Révoltes logiques, au nom tiré du poème Démocratie de Rimbaud, également écrit après celle$\mathrm{Ci}^{14}$.

Pour autant, l'explication par le seul biais de l'anticommunisme de cette nonréception de l'essai, comme des livraisons de la revue, paraît un peu courte. L'engagement militant des rédacteurs dans une mouvance maoïste toujours plus déliquescente vaut là chiffon rouge. Elle oblitère un sous-titre de l'essai plus éclairant, À propos del'histoire et des historiens, dont les propositions théoriques font écho à la controverse entre Les Révoltes logiques et Le Mouvement social en 1977, à l'occasion du $100^{\mathrm{e}}$ numéro de ce dernier, qui vaut en quelque sorte reconnaissance du travail de la revue dans la fondation d'une histoire scientifique de la classe ouvrière et du mouvement ouvrier: dans la ligne de mire des Révoltes logiques, une certaine pratique de l'histoire sociale. J e poserai alors comme postulat que cette mouvance décline un autre faire de l'histoire que les propositions académiques de la Nouvelle histoire, du Mouvement social. Dans l'ordre de la controverse, des critiques venues des marges militantes de la discipline prennent à partie des positions établies. On ne peut entendre ces pratiques dans leur seule dimension idéologique, pourtant contextuellement prégnante: elles impliquent, dans le temps court d'une épistémologie engagée, le recours à d'autres propositions sur les modalités de la pratique de l'histoire, comme sur la définition des frontières et des agrégats qui informent ces objets. Mon propos s'efforcera alors de définir ce passé dont il s'agit de faire table rase, pronostiquant qu'en la matière, il s'agit peu de biffer d'un trait la pratique de l'histoire, mais bien davantage de proposer d'autres modalités d'organisation du faire de l'histoire, ici et maintenant (1976) certes, mais par l'emprunt, le transfert, d'autres modèles épistémologiques.

${ }_{13}$ Marc Ferro, Mes histoires parallèles. Entretiens avec Isabelle Veyrat-Masson, Paris, Carnet-Nord, 2011, p. 190.

${ }^{14}$ J acques Rancière, « Les gros mots », op. cit., note 9. 
Vincent Chambarlhac, «Du passé faisons table rase?, un vandalisme épistémologique?», Histoire@Politique. Politique, culture, société, $\mathrm{n}^{\circ} 23$, mai-août 2014 [en ligne, www.histoirepolitique.fr]

\section{Un autre Faire de l'histoire}

J ean Chesneaux titre de manière interrogative son essai, ce que les rares recensions contemporaines omettent. Du passé faisons table rase? se présente comme une interrogation non de la discipline elle-même mais des modalités de sa pratique; l'ouvrage vaut manifeste du mouvement Forum-histoire. Celui-ci naît à Paris VII les 24 et 25 mai 1975; dès janvier 1976, il se dote des Cahiers du Forum-histoire, édités par Maspero à partir du sixième numéro (1978). L'interrogation de J ean Chesneaux procède de la remise en cause d'une pratique académique de l'histoire illustrée par la Nouvelle histoire. L'une des racines de l'ouvrage est sans doute la recension parue dans La Quinzaine littéraire le 1er décembre 1974 de Faire de l'histoire. L'article émane du «Collectif anti-Clio», auquel appartient J ean Chesneaux. Son titre vaut position militante dans le champ académique: «Entendent-ils les sommations du présent?». La recension promeut une autre définition de l'historien, de son rôle social, contre celle des auteurs de Faire de l'histoire :

« Les historiens devraient d'abord se définir par rapport à leur temps, regarder en face leur engagement social et ses implications pour le travail historique. Ce qui conduit à renoncer à un discours historique unifié et conciliateur, dans une société divisée. (...) Pour montrer à Le Goff, Nora, et leurs collègues qu'il existe une façon radicalement différente de faire de l'histoire, il faut dépasser la "pratique théorique" mise à la mode par les althussériens. Il faut passer plus de temps avec ceux qui "font l'histoire" au plein sens du mot, et un peu moins avec ceux qui se contentent de "faire de l'histoire"15. »

En écho, Les Révoltes logiques se définissent d'une manière quasi identique face à l'histoire pratiquée par Le Mouvement social :

«Ce qui nous intéresse: que les archives soient des discours, les "idées" des événements, que l'histoire soit en chaque instant rupture, questionnable seulement d'ici, seulement politiquement ${ }^{16}$. »

La parenté des propositions est étroite. Du « Collectif anti-Clio », nette préfiguration de Forum-histoire, au collectif des Révoltes logiques, un même fil rouge court : celui du gauchisme d'après-Mai 68 et de ses effets, repérés par l'historiographie, sur les sciences sociales ${ }^{17}$. L'horizon des analyses, principalement centré sur le Mai français, relègue au second plan les modalités d'organisation de ces collectifs. Elles déterminent pourtant la logique de cet autre « faire » de l'histoire proposé.

Forum-histoire et les Révoltes logiques ne se situent pas en marge de l'institution mais s'y inscrivent pleinement. Le premier naît à Paris VII, il regroupe des

15 Cf. Jean Chesneaux, «Entendent-ils les sommations du présent?», dans L'engagement des intellectuels. Itinéraire d'un franc-tireur, Toulouse, Privat, 2004, p. 185.

${ }_{16}$ Collectif Révoltes logiques, «Deux ou trois choses que l'historien social ne veut pas savoir », Le Mouvement social, $n^{\circ} 100$, juillet-septembre 1977, p. 30 .

17 François Dosse, « Mai 68, les effets de l'histoire sur l’Histoire », Politix, n 6, printemps 1989, p. $47-$ 52. Kristin Ross, Mai 68 et ses vies ultérieures, Bruxelles, Complexe/ Le Monde diplomatique, 2005. Vincent Chambarlhac, « Court voyage au pays des Révoltes logiques, ou d'une part de l'effet 68 sur les sciences sociales », Dissidences, $\mathrm{n}^{\circ} 5$, avril 2008. Voir également: " "Nous aurons la philosophie féroce."Les Révoltes logiques (1975-1981) », La Revue des revues, printemps 2013, op. cit. 
Vincent Chambarlhac, «Du passé faisons table rase?, un vandalisme épistémologique?», Histoire@Politique. Politique, culture, société, $\mathrm{n}^{\circ} 23$, mai-août 2014 [en ligne, www.histoirepolitique.fr]

universitaires comme des enseignants (du primaire et du secondaire) d'histoire; le second procède en partie de l'université de Vincennes au statut expérimental alors ${ }^{18}$. Comme tels, ces collectifs s'ancrent pleinement dans le champ disciplinaire historique, tout en se démarquant de son orientation vers une " histoire immobile ${ }^{19}$ » par le souci d'une pratique ouverte aux amateurs, déterminée par les questions du présent. La politisation du rapport à l'histoire participe de cette ouverture aux luttes du présent. Pour J ean Chesneaux, la forme du collectif participe du transfert sur la scène française des « radical caucuses » américains, qu'il définissait ainsi en 1970 :

« Un radical caucus, c'est un groupement, plus ou moins formellement structuré, d'intellectuels de gauche qui mènent la lutte politique et idéologique à l'intérieur d'une discipline universitaire donnée (histoire, sociologie, orientalisme) ${ }^{20}$. »

Forme du politique dans l'arène disciplinaire, le « radical caucus » participe de la génération étudiante contestatrice de la guerre du Vietnam aux États-Unis ${ }^{21}$. L'hypothèse d'un transfert culturel fonctionne alors, avec les spécificités propres à la scène française qu'illustre le Forum-histoire: doté de cahiers, le mouvement trouve son point d'appui à la charnière de l'enseignement et de la recherche tout en peinant à élargir sa base sociale hors du strict champ disciplinaire. Les déconvenues des rencontres du Forum-histoire avec les paysans du Larzac l'illustrent ${ }^{22}$. Dans le champ disciplinaire, et par homothétie avec le modèle des campus américain, Forumhistoire pratique une « histoire guérilla » en passant dans «le camp des luttes populaires » dont Du passé faisons table rase? constitue l'expression pamphlétaire aboutie. Ses flèches contre la Nouvelle histoire fusionnent deux logiques. La première tient à la dénonciation d'une pratique qui, selon les mots d'Emmanuel Le Roy Ladurie, entend séparer la recherche historique du politique ${ }^{23}$; la seconde procède de ce positionnement politique de Forum-histoire qui enjoint le questionnement du passé par le présent au titre du futur. Reçue du côté des règles de la discipline, la première de ces logiques participe du champ sémantique du vandalisme; l'histoire pratiquée par le Forum-histoire est étrangère aux règles $d u$ métier, son éventuel succès annoncerait la ruine de la science historique. Le silence fait sur la seconde dans l'immédiate réception de l'ouvrage oblitère ensuite le questionnement épistémologique porté24, appréciant l'essai et l'expérience qui le soutient sous le seul angle de l'idéologie, ici communiste dans sa version maoïste. Ce questionnement épistémologique, le collectif des Révoltes logiques permet de l’estimer plus précisément. La revue ne se donne jamais, fusse dans l’après-coup, de

18 Charles Soulié (dir.), Un Mythe à détruire? Origines et destin du Centre universitaire expérimental de Vincennes, Saint-Denis, Presses universitaires de Vincennes, 2012.

19 Il s'agit du titre de la leçon inaugurale d’Emmanuel Le Roy Ladurie au Collège de France le 30 novembre 1973.

20 J ean Chesneaux, «Le mouvement des "radical caucuses" », dans les sciences humaines aux ÉtatsUnis », L’homme et la société, $n^{\circ} 16, p .5$.

${ }^{21}$ Marie-Christine Granjon, "Révoltes des campus et nouvelle gauche américaine », Matériaux pour l'histoire de notre temps, 1988, n 11-13, p. 10-17.

22 « "Faire de l'histoire..." avec les paysans du Larzac (I et II) », Cahiers du Forum-histoire, n 5 et 6.

23 Emmanuel Le Roy Ladurie cité par François Dosse, L’histoire en miettes. Des «Annales » à la « Nouvelle histoire», Paris, La Découverte, 1987, p. 228.

24 Alban Bensa, Gérard Noiriel, «Le temps et l'histoire. Entretien avec J ean Chesneaux », Genèses, $\mathrm{n}^{\circ} 29,1997$, p. 123-140. 
Vincent Chambarlhac, «Du passé faisons table rase?, un vandalisme épistémologique?», Histoire@Politique. Politique, culture, société, $\mathrm{n}^{\circ} 23$, mai-août 2014 [en ligne, www.histoirepolitique.fr]

généalogie, sinon celle d'une naissance dans le contexte sartrien d'une histoire télévisuelle de la révolte avortée en 1974 , dont le projet l'enfante par ricochet ${ }^{25}$. A contrario, la revue consacre une longue recension en 1978 à l'expérience d'History Workshop au collège Ruskin ${ }^{26}$, qui éclaire en partie son projet. Initiée en $1967 \mathrm{au}$ collège Ruskin à Oxford, cette expérience se présente sous la forme d'ateliers annuels mêlant jeunes chercheurs, archivistes, enseignants, autodidactes et membres de sociétés d'histoire locale pour « écrire ensemble une autre histoire ${ }^{27}$ », qui donne naissance à un journal éponyme en 1975. Ce mode de fonctionnement abolit toute distinction entre « l'historien de métier » et l'historien « amateur » et ou « militant ». Implicitement l'ensemble de ces collectifs récusent la notion même d'historien professionnel quand, précisément, cette professionnalisation qui suppose la légitimité universitaire fonde alors l'histoire sociale, la dégondant de son âge militant. Le succès de l'history workshop tient à la fois à son enracinement local, et à la construction d'objets (les luttes populaires, le féminisme, etc.) ou à la déconstruction de catégories alors données comme évidentes (le mouvement ouvrier), dont le journal tôt mué en revue à l'écho international, assure la publicité. Le choix de cette recension dans les colonnes des Révoltes logiques implique, sinon la similitude des expériences (le collectif est plus resserré), l'identité du projet : soit l'attention portée à une histoire d'en bas, attentive aux acteurs, à la discontinuité et à la complexité des sources possibles pour cette pratique.

Par leurs projets respectifs, Forum-histoire et les Révoltes logiques s'inscrivent dans la mouvance de la New Left History hybridée ici par la dynamique politique postmaoïste à l'origine de ces collectifs. L'histoire qu'ils pratiquent se veut attentive aux luttes, aux exclus, aux marginaux ; elle emprunte nombre de ses traits à l'History from Below d'E. P. Thompson, et à son ouvrage The Making of the English Working class $(1963)^{28}$. Les racines intellectuelles de ces collectifs composent donc avec un canevas analytique marxiste (Gramsci notamment) revisité entre autres par la tradition empirique anglo-saxonne ${ }^{29}$, et attentif à la question foucaldienne des discours, des résistances, des micro-pouvoirs. Résolument située du côté des masses populaires en histoire, cette pratique questionne sans cesse par l'archive, dans l'histoire et par la réfraction du présent des luttes, les notions de conscience de classe, de lutte de classes, de révoltes... Parce qu'attentive à la question des masses, cette histoire par le bas que pratiquent ces collectifs rencontre le travail de la Nouvelle histoire comme de l'histoire sociale, questionnant les «mentalités populaires », les groupes sociaux. Cette rencontre suppose le heurt : aux « mentalités » de la Nouvelle

25 Vincent Chambarlhac, « Court voyage au pays des Révoltes logiques », op. cit., note 15.

${ }^{26}$ Andrew Lincoln, « History Workshop », Les Révoltes logiques, printemps-été 1978, p. 104-106.

${ }_{27}$ Christophe Topalov, « recension, à propos de Raphaël Samuel (ed.), History Workshop », Genèses, 1992, vol. 9, n 1, p. 170-171.

28 Jacques Rancière, dans la postface qu'il donne à la réédition de La parole ouvrière (Paris, La Fabrique, 2007) écrit : « (...) The Making of the English Working Class d’Edward P. Thomson offrait le modèle d'une histoire du combat ouvrier intégrant toutes les dimensions politiques, sociales et culturelles d'une expérience historique, en traitant les ouvriers non plus en corps souffrants mais en êtres parlants et pensants » (p. 338). Voir aussi l'introduction de François Jarrige à la réédition de l'ouvrage (Seuil, coll. « Points », Paris, 2012).

29 Peter Burke, " "Pas de culture je vous prie, nous sommes britanniques", l'histoire culturelle en Grande-Bretagne avant et après le tournant », dans Philippe Poirrier (dir.), L'Histoire culturelle : un tournant mondial del'historiographie?, Dijon, EUD, 2008, p. 16-25. 
Vincent Chambarlhac, «Du passé faisons table rase?, un vandalisme épistémologique?», Histoire@Politique. Politique, culture, société, $\mathrm{n}^{\circ} 23$, mai-août 2014 [en ligne, www.histoirepolitique.fr]

histoire, ces collectifs opposent une lecture en termes d'aliénation, de subversion que le travail de l'historien doit démasquer, dévoiler. Jacques Rancière confiait: « L'Histoire des mentalités me servait à la fois de modèle et de repoussoir ${ }^{30}$. » Sur un terrain de recherche historique similaire, ces collectifs se posent ainsi en challengers d'une écriture de l'histoire par la réflexion politique à l'origine de ce travail sur les luttes d'hier. C'est une épistémologie concurrente du travail des Annales comme de l'histoire sociale pratiquée par le Mouvement social qui se déploie aux marges du territoire del'historien.

\section{Une épistémologie vandale?}

À ce point du raisonnement, l'hypothèse d'une « épistémologie vandale » renseigne à nouveau les courtes citations des Annales. Celles-ci valent panorama pour embrasser l'arc des significations de ce « vandalisme épistémologique ». Il a évidemment partie liée avec l'immédiateté du lien présent des luttes/histoire ouvrière, supposant le militant dans l'historien.

$\mathrm{Au}$ plus près de l'orientation nouvelle des Annales, l'essai de Jean Chesneaux se reçoit d'abord comme la négation même du métier d'historien à suivre Emmanuel Le Roy Ladurie :

« Du constemant, passons au scandaleux : signant son propre acte de décès, et le nôtre en tant qu'historien, Chesneaux déclare froidement (p. 176) que le militant seul [sic], et non le chercheur, peut comprendre historiquement dans l'action un mouvement révolutionnaire (...).

Que le militant dispose de lumières précieuses sur sa propre conduite, je n'en doute pas. Mais que l'intellectuel doive se coucher totalement devant l'homme d'action, abdiquer sa faculté scientifique de réflexion, voilà qui est incroyable ${ }^{31}$. »

La charge est rude, mais ne surprend guère. Ici, il est d'autant moins de superpositions possibles que l'extrait sur lequel s'appuie Emmanuel Le Roy Ladurie figure dans le chapitre conclusif de l'essai de J ean Chesneaux, significativement titré «Quelle histoire pour la révolution ? », portant sur l'histoire immédiate. L'historien ne saurait être militant, les règles du métier l'imposent. À l'occasion de la table ronde organisée pour le $100^{\mathrm{e}}$ numéro du Mouvement social, cette déliaison ne va pas exactement de soi et dans les échanges entre J acques J ulliard, Maurice Agulhon, Guy Bourdé notamment, la lecture de l'ouvrage Du passé faisons table rase ? vaut balise :
« Moi aussi [J acques Julliard]. Pour beaucoup de camarades qui ont alors opéré la rupture avec une histoire sinon toujours hagiographique, du moins naïvement militante, donner un statut scientifique à l'histoire ouvrière constituait bel et bien une forme de contribution militante au mouvement ouvrier. Car l'histoire ouvrière souffrait d'une mauvaise réputation, chez les sociologues par exemple. On lui reprochait d'être le fait de "compagnons de route" nostalgiques et fort peu critiques. Mais en cours de route, les nouveaux historiens se sont pris au jeu : le souci scientifique a complètement oblitéré les motivations militantes initiales au point de les faire se désintéresser complètement de la destination sociale de leur travail. Il faudrait

30 J acques Rancière, Et tant pis pour les gens fatigués !, Entretien réalisé par Edmond El Maleh...», op. cit., note 10.

${ }^{31}$ Emmanuel Le Roy Ladurie, « J danov à Pékin », art. cit., note 3. 
Vincent Chambarlhac, «Du passé faisons table rase?, un vandalisme épistémologique?», Histoire@Politique. Politique, culture, société, $\mathrm{n}^{\circ} 23$, mai-août 2014 [en ligne, www.histoirepolitique.fr]

alors parler d'une troisième phase dans l'histoire de la revue, celle contre laquelle sont en réaction les gens comme Chesneaux. (... ${ }^{32}$. »

Chesneaux est, dans l'horizon de J acques Julliard, recevable en tant qu'il éclaire le chemin parcouru par l'histoire sociale que pratique la revue. Du passé faisons table rase? est l'effet d'une position militante dans l'institution, ou plus exactement d'une contribution militante au mouvement ouvrier par un historien qui ne s'est pas pris au jeu auquel il participe comme universitaire. Comme telles les propositions de J ean Chesneaux sont à la fois recevables et datées, puisque témoignant d'un âge préscientifique de l'histoire sociale. Guy Bourdé peut alors à la fois railler ce « petit livre vert » et reconnaître qu'il interpelle directement la revue ${ }^{33}$ en ses méthodes de travail fondées sur le sériel, le quantitatif, l'ordinateur. Ces courtes occurrences de l'ouvrage dans la table ronde éclairent le jugement des Annales. Au terme de son réquisitoire, Emmanuel Le Roy Ladurie concède la «roublardise » et la «naïveté » des propositions de J ean Chesneaux, qualités qui se relient à la présentation implicite de l'âge militant de l'histoire ouvrière. Celle-ci s'est d'abord écrite par des militants, dans la littérature grise des brochures et des biographies exemplaires, durant les années 1930 ; Edouard Dolléans, Georges Bourgin, notamment en constituent les protagonistes dans le cadre des dispositifs de formation syndicale. La naissance de l'Institut français d'histoire sociale à l'initiative de ces derniers, en compagnie de J ean Maitron et sous la houlette d'Ernest Labrousse (1949), annonce la mutation de cette pratique vers l'histoire sociale du mouvement ouvrier, à la scientificité plus assurée. La seconde génération de ce mouvement (J acques Julliard, Rolande Trempé, Michelle Perrot...) et la fondation du Mouvement social (1960) achèvent la mutation ${ }^{34}$. C'est alors parce que cet âge militant est révolu que la charge critique procède implicitement du vandalisme épistémologique. Devant la centralité d'une histoire ouvrière tout entière construite par la scientificité de l'histoire sociale, l'âge militant de cette historiographie n'est plus de mise. Les philippiques de J ean Chesneaux contre l'histoire sérielle participent par le rejet de l'ordinateur d'une forme de ludisme historiographique quand la négation de l'historien par le militant sous la plume de J ean Chesneaux vaut destruction de la profession par le politique. Guy Bourdé peut ainsi conclure à la fois sur le bien-fondé des objections de J ean Chesneaux à la pratique historique, sa scientificité, et simultanément déclarer qu'il ne propose « ni plus, ni moins, [que] le retour au jdanovisme, ou au présentisme, c'està-dire une bonne vieille doctrine qui a fleuri dans les écoles américaines autour des années 1930 ou 1940, et qui consiste à examiner les problèmes historiques à partir du présent ${ }^{35} \gg$.

\footnotetext{
32J acques J ulliard, dans « Histoire ouvrière, histoire sociale (table ronde) », Le Mouvement social, $\mathrm{n}^{\circ} 100$, juillet-septembre 1977, p. 52.

33 Guy Bourdé, ibid., p. 59. Il fait alors allusion au chapitre 15 « Histoire par en haut et histoire par en bas...» (J ean Chesneaux, Du passé faisons table rase?, op. cit., 1976, p. 138-147).

${ }^{34}$ Fabrice d'Almeida, Histoire et politique, en France et en Italie : l'exemple des socialistes (1945-1983), Rome, École française de Rome, 1998. Également, Xavier Vigna, « La construction d'une histoire du syndicalisme: 1945-1954. Édouard Dolléans et ses réseaux », Vincent Chambarlhac, "Lefranc et l'écriture de "l'explosion sociale" du Front populaire», dans colloque « Histoire et mémoire des mouvements syndicaux au XXe siècle : regards croisés sur le Puy-de-Dôme et la France », organisé à Clermont-Ferrand le 12 décembre 2012, à paraître.

35Guy Bourdé, ibid., p. 65.
} 
Vincent Chambarlhac, «Du passé faisons table rase?, un vandalisme épistémologique?», Histoire@Politique. Politique, culture, société, $\mathrm{n}^{\circ} 23$, mai-août 2014 [en ligne, www.histoirepolitique.fr]

« J danov est à Pékin » - à moins que ce ne soit Madison ${ }^{36}$ - , Clio ne saurait y résider. L'hypothèse d'un « vandalisme épistémologique » trouve là sa première condition de possibilité ; elle s'établit dans l'œil de l'institution contre celui - ceux - qui gomment la distinction des rôles et des écritures en son sein. Ce premier état de l'hypothèse se double de l'interrogation de la pratique de l'histoire proposée par ces collectifs en miroir des conditions de production du savoir historique comprises là comme un patrimoine, à protéger.

Le collectif des Révoltes logiques est là bien plus explicite que l'ouvrage Du passé faisons table rase?. Invité par le Mouvement social à discuter l'orientation de la revue, il affirme :

«Ce sera aussi bien de dire pourquoi, bien que nous fassions des recherches sur le même terrain (celui du dressage des travailleurs, des luttes ouvrières, de l'histoire militante ou des cultures populaires...) nous entendons ne pas faire d'histoire sociale ${ }^{37}$. »

Le « faire de l'histoire» de cette mouvance s'apprécie donc par la dénégation. La radicalité de ses propositions tient en quelques mots: l'historien social, sous l'apparente exhibition d'un savoir nouveau construit par la linguistique, l'informatique et le développement des sciences humaines, ne confirme en fait qu'un déjà su puisque l'ensemble des procédures choisies ignore la singularité au profit de la série, vise à s'inscrire dans un savoir perpétuellement cumulatif. Il y aurait là « [un] effet science qui revalorise le capital constant du savoir historique ${ }^{38}$ ». Cet effet science pour le collectif découle de la pratique de l'archive d'État par l'historien social ; «instance qui lui fournit le plus de renseignements», il en ignore la politique : celle d'une prise d'État sur l'individu, d'une mise en série par les dossiers constitués, d'un enrôlement dans un récit déjà là, ourdit par l'administration en ses fonctions régaliennes et répressives qui, ensuite, devient matériau de l'analyse historique. Le collectif peut alors conclure sur l'histoire sociale, sur ses buts :

«L'impensé d'un objet, les frontières d'une discipline, les échelons d'un cursus ferment le cercle de cette histoire. (...) Ce qui nous intéresse, que les archives soient des discours, les "idées" des événements, que l'histoire soit en chaque instant rupture, questionnable seulement d'ici, seulement politiquement ${ }^{39}$. »

Tout concourt alors à ce que l'affirmation du présent politique des questions posées fasse table rase des interprétations antérieures. Le vandalisme épistémologique de ces collectifs tient finalement peu à l'histoire comme objet et davantage aux praticiens qui assurent la continuité d'analyses à grands renforts de méthodologies renouvelées, qui toutes omettent la réflexion sur ce que l'archive recèle de singulier, d'irrégulier, d'intempestif, d'altérité. Jacques Rancière peut a posteriori dater l'événement fondateur de cette nouvelle épistémologie, Lip en 1973 :

\footnotetext{
36 J ean Chesneaux rédige le manifeste de Forum-histoire avec l'historien américain Harvey Goldberg, signant tous deux sous le pseudonyme de Tenney-Park (Madison, Wisconsin) où enseigne Goldberg, où se manifestait en histoire le phénomène des radical caucuses. Cf. J ean Chesneaux, L'engagement des intellectuels, op. cit., note 7, p. 185.

${ }^{37}$ Collectif Révoltes logiques, Deux, trois choses que l'historien social ne veut pas savoir, op. cit., note 8, p. 21.

38 Ibid., p. 27.

39 Ibid, p. 30.
} 
Vincent Chambarlhac, «Du passé faisons table rase?, un vandalisme épistémologique?», Histoire@Politique. Politique, culture, société, $\mathrm{n}^{\circ} 23$, mai-août 2014 [en ligne, www.histoirepolitique.fr]

«L'initiative des Lip ramenait au premier plan l'idée d'une tradition autonome de lutte ouvrière, refusant de séparer l'économie et la politique, la lutte revendicative et l'association ouvrière de production, le combat présent et l'anticipation d'un monde à venir. Elle invitait à reconsidérer des traditions ouvrières battues en brèche par la vision stratégique du marxisme et par sa stricte séparation entre la lutte économique et la lutte politique: celle de l'association ouvrière et du syndicalisme révolutionnaire ${ }^{40}$. »

La citation affine notre propos. S'il est un vandalisme épistémologique, il tient à l'irruption sur la scène des luttes ouvrières d'acteurs auparavant invisibles dans les récits de ces luttes. Leur seule apparition autorise l'insubordination d'une partie des producteurs et des diffuseurs du savoir historique contre l'édifice disciplinaire.

L'argument d'un «vandalisme épistémologique » fait évidemment violence à ces textes et à ces prises de position. Son emploi vaut pourtant clé heuristique pour saisir le grondement des batailles qui accompagnait naguère la centralité de l'histoire ouvrière et sociale. Au défaut de ces affrontement, les âges militant et scientifique d'une histoire sociale glissant vers les mentalités, les représentations, en ce milieu des années 1970 et l'idée, finalement, que dans la réception de J ean Chesneaux la tabula rasa peut aussi s'entendre comme l'incongruité de l'association du militantisme à l'histoire ouvrière, dont pourtant elle procédait, naguère. La réception de la thèse de Jacques Rancière, La Nuit des prolétaires, en 1981 affine le constat. Si ce livre s'entend comme une conclusion à l'aventure intellectuelle collective des Révoltes logiques, il ne retient pas l'attention du Mouvement social. Tout juste est-il mentionné dans la rubrique des livres reçus. Pour l'auteur, le projet du livre permettait, en philosophe, « une interrogation sur la fonction critique conférée à l'histoire, sur le rôle présent de l'historien dans notre culture. Il est celui qui "démystifie", qui renvoie les illusions de la subversion gauchiste aux conditions matérielles et aux comportements qu'elles autorisent. Mais cette fonction critique se double d'une production d'évidence plus dogmatique au fond que les idéologies ${ }^{41} \gg$.

Cette interrogation ne retient donc pas l'attention de l'histoire sociale, de la Nouvelle histoire, bien que les Annales évoquent brièvement l'ouvrage dans « Le choix des Annales », notant sa contribution à « l'histoire des singularités qui s'insinue au coeur de la plus nouvelle des histoires ${ }^{42}$ ». La remarque induit un regard bienveillant, tout en rétrécissant l'ouvrage à sa seule étude d'itinéraires de prolétaires saint-simoniens. C'est sur les marges de l'histoire, dans le champ de l'histoire de l'éducation, que Michelle Perrot publie une longue recension de La Nuit des prolétaires, s'efforçant de situer son enjeu devant l'histoire de la lecture, de l'écriture, de l'autodidaxie. Au cour de son propos, la notion d'« écrivant »43. Mais l'histoire culturelle est encore en devenir. Le silence fait sur La nuit des prolétaires dans le champ de l'histoire sociale, hors ces deux recensions, atteste d'une réception réduite aux acquêts, l'ouvrage

\footnotetext{
40 J acques Rancière, La parole ouvrière, op. cit., note 19, p. 337.

41 J acques Rancière, «Et tant pis pour les gens fatigués! Entretien réalisé par Edmond El Maleh...», op. cit., note 10 , p. 36.

42 Annales. Économies, Sociétés, Civilisations, 36e année, n 3, 1981, p. 528.

43 Michelle Perrot, « Note critique à propos de Jacques Rancière, La nuit des prolétaires. Archives du rêve ouvrier, Paris, Fayard, 1981 », Histoire de l'éducation, ${ }^{\circ}$ 13, 1981, p. 80-83.
} 
Vincent Chambarlhac, «Du passé faisons table rase?, un vandalisme épistémologique?», Histoire@Politique. Politique, culture, société, $\mathrm{n}^{\circ} 23$, mai-août 2014 [en ligne, www.histoirepolitique.fr]

s'imposera plus tard avec le développement de l'histoire culturelle du politique, et l'affirmation d'une réflexivité de l'historien face à ses objets, à sa pratique ${ }^{44}$.

Pour autant la dynamique épistémologique de ces collectifs comme Forum-histoire ou les Révoltes logiques, nés du post-maoïsme qui ne survivront pas à ce temps de restauration qu'est l'entre-deux-mai, imprime sa marque aux pratiques contemporaines d'une histoire sociale et culturelle.

Quelques signes l'attestent. Au premier chef, la question posée par l'irruption du postcolonialisme : Can the Subaltern Speaks ? L'interrogation reprend, sur le mode d'une histoire globale et connectée, l'antienne militante des collectifs des années 1970. Elle est moins l'expression d'une apostrophe politique que la marque d'une interrogation du regard porté sur les sources et leur lecture. Son acclimatation par des collectifs à la jointure du militantisme et de la recherche ${ }^{45}$ peut donc trouver des antécédents dans ces propositions des années 1970.Christophe Prochasson dans L'empire des émotions pourrait reprendre à son compte une part de la charge d'Emmanuel Le Roy Ladurie, transposée à la scène contemporaine des usages des Cultural Studies, et partager les réserves de Jacques Julliard sur engagement et pratique de l'histoire. On retrouve dans un questionnement tramé par une contemporanéité politique et radicale un égal rapport à une pratique de l'histoire qu'il s'agit peu d'écrire au prisme des vaincus mais bien davantage à l'aune d'une réalité contemporaine dont il s'agirait d'éprouver le temps long. Dans les philippiques du court essai de Jean-François Bayart - un carnaval académique - sur le postcolonialisme $^{46}$ se rejouerait donc une scène déjà visitée lors des années 1970, celle du rapport de l'histoire sociale au politique, celle surtout des rapports du chercheur critique à la profession d'historien. Celle donc du rôle social de l'historien. $\mathrm{Si}$ « le bon historien est l'ogre de la légende », flairant la chair humaine, devinant là son gibier comme l'indiquait Marc Bloch dans Apologie pour l'histoire, alors cette disposition l'expose à l'interrogation toujours renouvelée de son rapport au politique.

\section{L'auteur}

Vincent Chambarlhac est maître de conférences en histoire contemporaine à l'université de Bourgogne, CGC CNRS 7366. Il a publié dernièrement avec Thierry Hohl, Un moment antifasciste (1934-1936), à La Ville brûle.

\section{Résumé}

La réception du livre de Jean Chesneaux (Du passé faisons table rase ?) par les historiens français débouche sur le heurt de deux manières de pratiquer l'histoire du mouvement ouvrier. L'une, académique, oppose à l'autre sa radicalité, son engagement. Celui-ci participerait d'un vandalisme épistémologique. Revenir sur cette

\footnotetext{
44 J ean-Pierre Rioux et J ean-François Sirinelli, Pour une histoire culturelle, Paris, Seuil, 1997.

45 Vincent Chambarlhac, «Fragments du jeu académique postcolonial (à propos d'un collectif, l'Association pour la connaissance de l'histoire de l'Afrique contemporaine, ACHAC) », Histoire@Politique. Politique, culture, société, $\mathrm{n}^{\circ} 12$, septembre-décembre 2010, www.histoirepolitique.fr

46 Vincent Chambarlhac, «Fragments du jeu académique postcolonial (à propos d'un collectif, l'Association pour la connaissance de l'histoire de l'Afrique contemporaine, ACHAC) », op. cit.
} 
Vincent Chambarlhac, «Du passé faisons table rase?, un vandalisme épistémologique?», Histoire@Politique. Politique, culture, société, $\mathrm{n}^{\circ}$ 23, mai-août 2014 [en ligne, www.histoirepolitique.fr]

polémique restitue une part du paysage intellectuel de l'histoire dans la décennie 1970.

Mots clés : histoire, épistémologie, extrême gauche, controverse.

\section{Abstract}

The reviews of J ean Chesneaux's book (Du passé faisons table rase?) by the French historians attest of two opposite ways of conceptualizing and making the history of the labour movement. The academic one stands against another one, characterized by its radicality and its political commitment, by its epistemological vandalism. The article highlights the intellectual controversy between The Annales and the Révoltes logiques group, and therefore draws the intellectual landscape of the history discipline in the 1970 s.

Keyword:History; Epistemology;Extrême-Gauche ; Controversy.

Pour citer cet article : Vincent Chambarlhac, «Du passé faisons table rase ?, un vandalisme épistémologique? », Histoire@Politique. Politique, culture, société, $n^{\circ} 23$, mai-août 2014 [en ligne, www.histoire-politique.fr] 